\title{
Prognostic Value of Stress Hyperglycemia in Patients Admitted to Medical/Geriatric Departments for Acute Medical Illness
}

\author{
Raffaella Di Luzio - Rachele Dusi - Francesca Alessandra Barbanti • \\ Pietro Calogero · Giulio Marchesini (1) · Giampaolo Bianchi
}

Received: August 24, 2021 / Accepted: November 17, 2021 / Published online: December 2, 2021

(c) The Author(s) 2021

\begin{abstract}
Introduction: Hyperglycemia is common in patients admitted to Italian medical/geriatric units and is associated with a poorer outcome. We tested the significance of diabetes and stressinduced hyperglycemia in clinical outcome.

Materials and Methods: Three hundred seventy-eight consecutive patients with hyperglycemia at entry $(\geq 126 \mathrm{mg} / \mathrm{dl})$ (206 without known diabetes) were included, with a wide range of underlying diseases requiring hospital admission and independent of the presence of diabetes. Relative hyperglycemia was calculated
\end{abstract}

Supplementary Information The online version contains supplementary material available at https:// doi.org/10.1007/s13300-021-01183-y.

R. Di Luzio

Unit of Internal Medicine and Diabetology, Local

Health Unit, Modena, Italy

R. Di Luzio · R. Dusi · F. A. Barbanti - P. Calogero ·

G. Bianchi $(\bowtie)$

IRCCS-Sant'Orsola-Malpighi Hospital, Bologna, Italy

e-mail: giulio.marchesini@unibo.it

P. Calogero

Department of Hospital and Community Medicine, Malpighi Hospital, Bologna, Italy

G. Marchesini - G. Bianchi

Department of Medical and Surgical Sciences, Alma Mater University, 9, Via Massarenti, 40138 Bologna, Italy as admission glucose divided by average glucose, estimated based of glycosylated hemoglobin. Values $\geq 1.20$ were considered indicative of stress hyperglycemia (SHR). The association of SHR with outcome variables (all-cause complications, infections, non-infectious events, deaths) was tested by logistic regression analysis, adjusted for sex, BMI, age-adjusted comorbidities (Charlson index) and known diabetes.

Results: During hospital stay, one or more events were registered in 96 patients (25.4\%); 44 patients died in hospital, and fatality rate was borderline higher in patients without diabetes $(14.6 \%$ vs. $8.1 \%$ in diabetes; $P=0.052)$ and nearly three times higher in patients with stress hyperglycemia (15.0\%) vs. those with SHR $<1.2$ $(P=0.005)$. Stress hyperglycemia-more common in the absence of diabetes (71\% vs. $58 \%)$ and age were the only independent prognostic factors for death. At multivariable analysis, the risks of death (OR 4.31, 95\% CI 1.25-14.81), of all complications (OR 5.90, 95\% CI 2.22-15.71) and of newly developed systemic infections (OR 5.67, 95\% CI 1.61-19.92) were associated with stress hyperglycemia in subjects without diabetes, as was the risk in non-insulin-treated cases (OR 4.02, 95\% CI 1.16-13.92; OR 5.47, 95\% CI 2.21-13.52; OR 5.15, 95\% CI 1.70-15.62, respectively).

Conclusion: The study confirms the prognostic value of stress-related hyperglycemia in patients requiring hospital admission to a geriatric/ medical unit for a variety of acute medical 
conditions, contributing to adverse outcomes not limited to events commonly associated with hyperglycemia (e.g., infections).

Keywords: Cardiovascular outcomes; Complications; Death; Diabetes; Hospital admission; Infection; Prognosis; Stress hyperglycemia

\section{Key Summary Points}

Why carry out this study?

Stress hyperglycemia has been associated with poor outcome in surgical settings and in patients admitted for cardiovascular disease

The significance of stress hyperglycemia in the general population of patients admitted to a medical/geriatric department, with a high prevalence of diabetes, has never been documented

The study tested the association of stress hyperglycemia with all-cause complications, infections and noninfectious events, and deaths in consecutive patients to clarify its prognostic role

\section{What was learned from the study?}

The presence of stress hyperglycemia, particularly in subjects without known diabetes, increased complication and death rates

The study confirms the prognostic value of stress hyperglycemia at hospital admission, which might be considered a concerning signal for patients in the medical/geriatric setting

\section{INTRODUCTION}

Hyperglycemia is common in patients at hospital admission in daily practice, not limited to patients with diabetes, but extending to individuals without a known history of diabetes. Most recent estimates suggest that its prevalence may be in the range of $25-35 \%$ in Italian patients admitted to medical/geriatric units [1-4], given the increase of diabetes prevalence with advancing age and comorbidities. Previously unknown, newly detected diabetes contributes to this prevalence [3], as well as stressinduced hyperglycemia $[2,5-7]$, driven by the acute response to clinical conditions requiring hospital admission. In these conditions, hyperglycemia is largely mediated by glucagon [8] in the presence of a previously normal glucose metabolism, in turn promoting oxidative stress and insulin resistance $[9,10]$.

Whatever the origin of hyperglycemia, it is associated with poor outcomes and prolonged hospital stay (increased case fatality rate and incidence of infections and further disability after hospital discharge in different settings $[6,7,11-13])$. There is compelling evidence that prompt treatment of any hyperglycemic state may help reduce adverse events, and in-hospital protocols for the implementation of basal-bolus regimens in the presence of hyperglycemia are advocated by national and international guidelines [14-16]. These regimens, which may be effectively managed by nurses with minimum impact on physicians' involvement [17], are aimed at achieving a rapid control of glucose levels with minimal risk of hypoglycemia compared with sliding-scale insulin treatment. Sliding-scale protocols continue to be largely in use outside diabetes units [18] because of their simplicity, although their use is discouraged by guidelines. In subjects with mild hyperglycemia, also the use of non-insulin agents might be preferred to intensified insulin to facilitate treatment and avoid the risk of hypoglycemia, but no safe values for glucose levels have ever been defined.

We aimed to determine the prevalence of stress-induced hyperglycemia in a large cohort of patients admitted to a geriatric department, independent of the presence of a diagnosis of diabetes, as well as the importance of glucose control and insulin treatment in the immediate management of hyperglycemia to reduce 
Table 1 Characteristics of the study population, grouped by the presence of diabetes

\begin{tabular}{|c|c|c|c|c|}
\hline Variable & $\begin{array}{l}\text { All cases } \\
(n=378)\end{array}$ & $\begin{array}{l}\text { Without DM } \\
(n=206)\end{array}$ & $\begin{array}{l}\text { With DM } \\
(N=172)\end{array}$ & $P$ value \\
\hline Age (years) & $78.3 \pm 12.4$ & $79.2 \pm 12.9$ & $77.3 \pm 11.7$ & 0.128 \\
\hline Age class $(<65 ; 65-65 ; 75-85 ;>85)(\%)$ & $14 / 16 / 36 / 34$ & $14 / 12 / 36 / 38$ & $15 / 20 / 35 / 30$ & 0.143 \\
\hline Male sex (\%) & $\begin{array}{l}47.6 \\
{[42.5-52.5]}\end{array}$ & $47.6[40.6-54.1]$ & $47.7[40.0-54.8]$ & 0.163 \\
\hline BMI $\left(\mathrm{kg} / \mathrm{m}^{2}\right)$ & $28.0 \pm 5.5$ & $27.0 \pm 5.2$ & $28.8 \pm 5.6$ & 0.117 \\
\hline $\begin{array}{l}\text { BMI class (normal, overweight, obesity } 1 \text {, obesity } 2 \text {, } \\
\text { obesity 3) (\%) }\end{array}$ & $30 / 35 / 24 / 11$ & $37 / 33 / 25 / 5$ & $24 / 37 / 24 / 15$ & 0.306 \\
\hline Smoke (no, present, ex) (\%) & $68 / 22 / 10$ & $71 / 19 / 10$ & $63 / 27 / 9$ & 0.126 \\
\hline Age-adjusted Charlson's score & $6.50 \pm 2.55$ & $6.61 \pm 2.22$ & $7.57 \pm 2.51$ & $<0.001$ \\
\hline Hemoglobin $(\mathrm{g} / \mathrm{dl})$ & $12.9 \pm 2.2$ & $13.1 \pm 2.2$ & $12.6 \pm 2.1$ & 0.014 \\
\hline Leucocyte count $(\times \mu \mathrm{l})$ & $11.1 \pm 5.5$ & $11.5 \pm 5.8$ & $10.7 \pm 5.1$ & 0.177 \\
\hline Platelets $(\times \mu \mathrm{l})$ & $238 \pm 101$ & $252 \pm 105$ & $219 \pm 97$ & 0.432 \\
\hline Creatinine $(\mathrm{mg} / \mathrm{dl})$ & $1.33 \pm 0.84$ & $1.31 \pm 0.87$ & $1.35 \pm 0.81$ & 0.601 \\
\hline $\mathrm{eGFR}\left(\mathrm{ml} / \mathrm{min} \times 1.73 \mathrm{~m}^{2}\right)$ & $61.6 \pm 30.8$ & $63.4 \pm 30.4$ & $59.5 \pm 31.3$ & 0.230 \\
\hline Potassium $(\mathrm{mEq} / \mathrm{l})$ & $4.18 \pm 0.71$ & $4.11 \pm 0.71$ & $4.26 \pm 0.71$ & 0.037 \\
\hline Total cholesterol (mg/dl) & $168 \pm 48$ & $170 \pm 45$ & $166 \pm 51$ & 0.440 \\
\hline HDL cholesterol $(\mathrm{mg} / \mathrm{dl})$ & $42.4 \pm 13.9$ & $44.1 \pm 14.6$ & $40.7 \pm 13.0$ & 0.050 \\
\hline Triglycerides $(\mathrm{mg} / \mathrm{dl})$ & $136 \pm 80$ & $123 \pm 70$ & $150 \pm 88$ & 0.005 \\
\hline Blood glucose at admission $(\mathrm{mg} / \mathrm{dl})$ & $192 \pm 90$ & $159 \pm 35$ & $230 \pm 118$ & $<0.001$ \\
\hline Glycosylated HbAlc (\%) & $6.51 \pm 1.59$ & $5.67 \pm 0.34$ & $7.51 \pm 1.89$ & $<0.001$ \\
\hline Relative hyperglycemia (\%) & $1.40 \pm 0.58$ & $1.43 \pm 0.66$ & $1.36 \pm 0.47$ & 0.297 \\
\hline Stress hyperglycemia $(\mathrm{RH}>1.2)(\%)$ & $\begin{array}{l}65.1 \\
{[60.0-69.6]}\end{array}$ & $71.4[64.6-76.9]$ & $57.6[49.8-64.4]$ & 0.007 \\
\hline Hospital length of stay (days) & $10.6 \pm 7.0$ & $9.8 \pm 6.2$ & $11.5 \pm 7.7$ & 0.019 \\
\hline
\end{tabular}

Data are reported as mean \pm SD or as percent [95\% confidence interval]

The diagnosis of diabetes was either retrieved from history or was based on glycosylated hemoglobin levels $\geq 6.5 \%$ ( $48 \mathrm{mmol} / \mathrm{mol}$ ) at time of admission (newly diagnosed diabetes)

eGFR estimated glomerular filtration rate (CKD-EPI) [29] 
hospital stay and the development of complications.

\section{METHODS}

\section{Patients}

The study was prospectively carried out in two medical/geriatric units of the Sant'Orsola-Malpighi Hospital, a university hospital with nearly 1000 beds, and in a peripheral hospital of the Local Health Unit, Bologna, Italy, from May 2017-September 2018. All patients aged $>18$ years, with hyperglycemia at entry $(\geq 126 \mathrm{mg} /$ dl) and providing informed consent to participation, were considered, independent of the reason(s) for admittance and the presence of diabetes. Their baseline data are reported in Table 1. Exclusion criteria were the presence of an active cancer disease, the recent use of steroids (orally or by injection), the use of parenteral nutrition and recent hemorrhage or blood transfusion (within the last 3 months). Data were collected at entry, and metabolic control, including insulin administration whenever needed, was monitored in the course of the first 6 days of hospital stay. Finally, the time to hospital discharge (or death) was recorded. In the presence of high blood glucose, treatment was based on a pre-planned protocol of insulin administration based on a basal bolus regimen, but in a few cases a common slidingscale intervention was implemented. This occurred because no shared protocols for insulin treatment are operative between the medical/geriatric units of the Sant'Orsola-Malpighi Hospital and the Local Health Unit of Bologna. The cut-off for insulin treatment was set at glucose values $\geq 200 \mathrm{mg} / \mathrm{dl}$ on admission. Considering the severity of condition and patients' frailty, the glucose target was set in the range $120-150 \mathrm{mg} / \mathrm{dl}$ to limit the risk of hypoglycemia.

The Case Report Forms were filled out by physicians soon after admittance and completed for missing elements after interviewing relatives. Specific care was taken in the assessment of the previous history of diabetes $(n=154)$, the use of glucose-lowering drugs and the presence of diabetes-related micro- and macro-vascular complications. This cohort also comprised three cases diagnosed as type 1 diabetes mellitus. Patients with glycosylated A1c hemoglobin levels $\geq 6.5 \% \quad(48 \mathrm{mmol} / \mathrm{mol})$ $(n=18)$ were later added to the cohort of diabetes as unknown diabetes. A total of 45 cases were treated solely by diet (26\%), $42 \%$ were on oral or non-insulin injectable glucose-lowering agents, $19 \%$ were being treated by insulin (basal or intensified insulin treatment) and 13\% received a combination of non-insulin and basal insulin treatment. From patient history we calculated the age-adjusted Charlson Comorbidity Index [19].

The protocol was approved by the ethics committee, Sant'Orsola-Malpighi Hospital (protocol 27/2017/U/Oss and amendments) and by the ethics committee of the Local Health Unit of Bologna (CE-BI 17044). All patients signed an informed consent to participation and data upload.

\section{Methods}

Body mass index (BMI) was calculated by weight and height, measured to the nearest $\mathrm{kg}$ and $\mathrm{cm}$ on a scale in light clothing. Laboratory values including glycosylated HbA1c levels were obtained by standard techniques on the day following admission by the Laboratorio Unico Metropolitano, a laboratory serving the whole area of Bologna and part of a program for the standardization of biochemical assays.

HbA1c was used to detect an unknown diabetic state $(\mathrm{HbA} 1 \mathrm{c} \geq 48 \mathrm{mmol} / \mathrm{mol})$ [2] as well as to estimate the average blood glucose concentration before admission using the equation: estimated average glucose $(\mathrm{mg} / \mathrm{dl})=\left(28.7^{*}\right.$ HbA1c [\%]) - 46.7 [20]. Using diabetes history and $\mathrm{HbA1c}$ as indicators, we grouped patients into three categories: (1) hyperglycemia at admission, no diabetes; (2) diabetes by history; (3) newly diagnosed diabetes. Their comorbidities are reported in Fig. 1 (upper panel). In particular, there was a high prevalence of hypertension $(76 \%)$ and cardiovascular disease (42\%) and arrythmias (30\%), but also chronic 


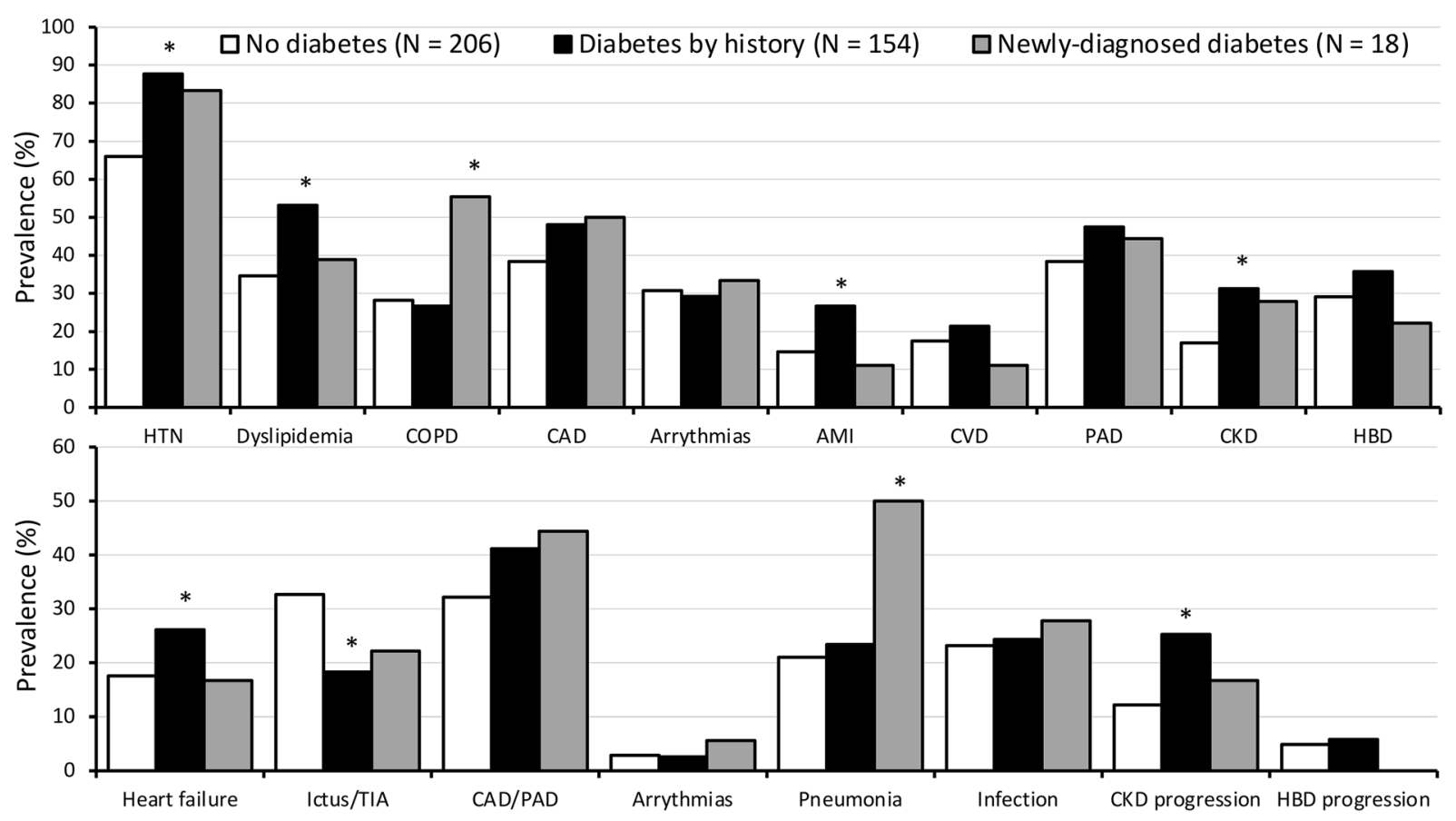

Fig. 1 Comorbidities at history in the study population (upper panel) and reasons for hospital admission and inhospital complications (lower panel), in relation to diabetes by history and diabetes diagnosed at admission. *Significant difference compared with the population without diabetes. HTN hypertension; COPD chronic

kidney disease (23\%) and hepatobiliary disease (26\%).

Relative hyperglycemia was defined by SHR, calculated as admission glucose divided by estimated average glucose [21]. The critical SHR value of 1.20 was considered indicative of stressinduced hyperglycemia, considering the uncertainty about adequate fasting time in a few patients. Accordingly, the categories were further divided according to the SHR cut-off to test the possible incremental role of stress hyperglycemia on the risk of complications, independent of diabetes.

The events requiring hospital admission, as well as the different comorbidities assessed by laboratory and imaging techniques, were recorded (Fig. 1, lower panel). Most cases were admitted for cardiovascular (36\%) and cerebrovascular disease $(27 \%)$, heart failure $(21 \%)$ and pneumonia (23\%), but newly developed infections (16\%) and progression of renal (18\%) obstructive pulmonary disease; $C A D$ coronary artery disease; $A M I$ acute myocardial infarction; $C V D$ cerebrovascular disease; $P A D$ peripheral artery disease; $C K D$ chronic kidney disease grade $3 \mathrm{~b}$ or higher; $H B D$ hepatobiliary disease

and hepatobiliary disease $(5 \%)$ were recorded during follow-up. The time course of blood glucose was regularly checked during the first 6 days or until normalization, as was the amount of insulin administered according to basal-bolus or sliding-scale protocols.

\section{Statistical Analysis}

The sample size was determined on the assumption that (1) stress hyperglycemia might be demonstrated in 50\% of the population with hyperglycemia at admission (independent of the presence of diabetes) and (2) hyperglycemia might treble the risk of events, including the primary outcome of death rate, considering the expected high prevalence of patients with cerebrovascular events and systemic infection in the participating units $[22,23]$. According to these assumptions, the sample size was 
determined in 380 patients and fixed at 400 to account for missing data. The protocol was however stopped in September 2017 because of transfer of the principal investigator to a different hospital.

Statistical analysis was performed using StatView 5.0 ${ }^{\mathrm{TM}}$ (SAS Institute Inc., Cary, NC) and SPSS for Windows v.21 (SPSS Inc., Chicago, IL, USA). Continuous variables were reported using mean and standard deviation (SD) or median and interquartile range (IQR), as appropriate. Absolute prevalence and percentage were used for categorical variables (dichotomous variables). Differences between continuous variables for the different cohorts were tested for significance using the MannWhitney $U$ test. Prevalence in different groups/subgroups was compared by chi-squared test or by Fisher's exact test when appropriate.

A logistic regression analysis was performed to ascertain the association of stress hyperglycemia with the outcome variables (death, allcause complications, newly developed systemic infection) after adjusting for sex, BMI, age-adjusted comorbidities (age-adj Charlson comorbidity index-CCI) [24] and the presence of diabetes. The presence of infection at admission was also considered and tested as confounder [25]. The relative risk of outcome variables was calculated as odds ratios (OR) and 95\% confidence interval (CI). In a final model, the protocol of insulin treatment (basal bolus vs. sliding scale) was added as independent variable. Finally, the role of factors potentially affecting HbA1c value (anemia, advanced kidney disease, cirrhosis) were tested as additional confounders [26-28]. A sensitivity analysis was also performed, using the lower SHR cut-off of 1.14, tested in a different setting [7], and the corresponding higher value of 1.26.

$P$ values $<0.05$ were considered statistically significant.

\section{Compliance with Ethics Guidelines}

The protocol was approved by the ethical committee, Sant'Orsola-Malpighi Hospital (protocol 27/2017/U/Oss and amendments) and by the ethics committee of the Local Health
Unit of Bologna (CE-BI 17044). All patients signed an informed consent to participation and data upload. This study was performed in accordance with the Helsinki Declaration of 1964 and its later amendments.

\section{RESULTS}

\section{Baseline Characteristics}

The whole population was elderly and overweight (Table 1), with $70 \%$ of participants $>75$ years and in the overweight/obesity range (without differences in relation to diabetes). Biochemistry was characterized by high white cell count, moderate renal impairment and differences in lipid profile (higher cardiovascular risk in the presence of diabetes). This last condition was confirmed by the analysis of comorbidities at history (Fig. 1, upper panel) or confirmed during hospital stay (Fig. 1, lower panel). Notably, more cases were classified as chronic kidney disease (CKD) grade $3 \mathrm{~b}$ or higher [29] in the population with diabetes. Length of stay was nearly 2 days longer in the presence of diabetes. Potassium levels were lower in diabetes and below the lower limit of normal $(3.6 \mathrm{mEq} / \mathrm{l})$ in $17.6 \%$ of total cases $(26.5 \%$, $10.3 \%$ and $6.3 \%$, in the absence of diabetes, in diabetes by history and in newly detected diabetes, respectively).

Stress hyperglycemia was very common at entry (nearly two thirds of the entire population) and more common in the absence of diabetes ( $71 \%$ vs. $58 \%)$. In the group with diabetes, it was detected in $58 \%$ of cases with a history of diabetes and in $50 \%$ of newly diagnosed cases.

\section{Hyperglycemia Treatment and Time Course of Blood Glucose}

Insulin treatment was instituted soon after entry in 180 cases; 5 patients with and 16 without history of diabetes did not receive immediate insulin in the presence of hyperglycemia $>200 \mathrm{mg} / \mathrm{dl}$, as indicated in the protocol. One was later diagnosed as newly discovered diabetes. Insulin was maintained 


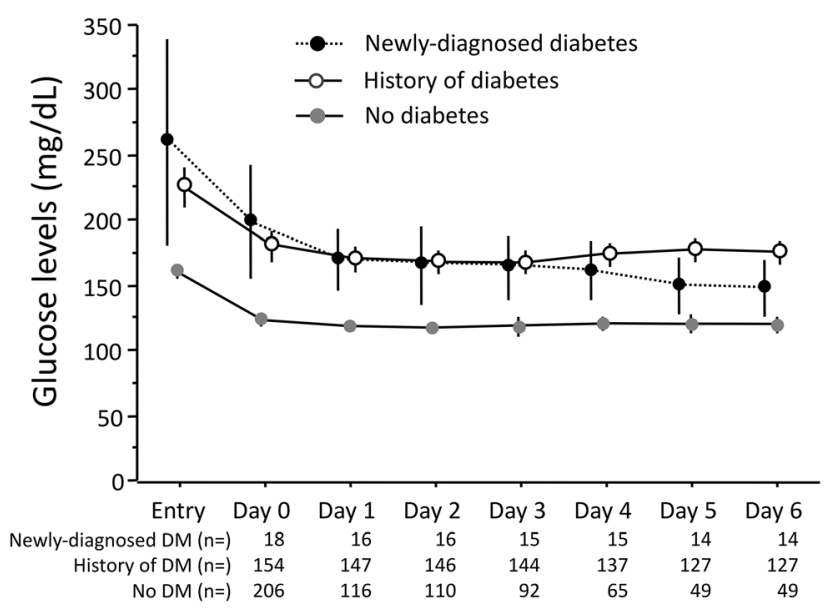

Fig. 2 Time course of blood glucose in the whole population, grouped according to presence of diabetes. The daily blood glucose represents the average of values

until death or discharge from hospital in 142 cases with diabetes (82.5\%) and only 20 with hyperglycemia without a previous diagnosis of diabetes (9.7\%). The average blood glucose level rapidly declined in all groups, but remained remarkably above the desired targets, particularly in the presence of diabetes (Fig. 2). The preplanned glucose target of $120-150 \mathrm{mg} / \mathrm{dl}$ was reached before death or discharge from hospital in all but one case without diabetes, in $81 \%$ with newly developed diabetes, but only in $64 \%$ with a history of diabetes. Basal insulin was used (and maintained) in six cases with newly diagnosed diabetes; oral hypoglycemic agents were progressively added in 61 cases with diabetes by history and in 6 cases with newly detected diabetes and also maintained after discharge from hospital.

Insulin infusion (always coupled with $\mathrm{K}^{+}$ administration) was only used in patients with diabetes as initial treatment (11 cases with known diabetes, 2 cases with newly developed diabetes). The majority of insulin-treated cases were managed according to a basal-bolus protocol (90 cases, 55.6\%), previously defined and implemented in the participating units, but several cases were treated according to a slidingscale procedure, more commonly used in subjects without diabetes $(n=22,10.8 \%)$ or in newly diagnosed diabetes $(n=8,44.4 \%)$ vs. measured in the course of the day. The progressive decline of study sample reflects both in-hospital deaths and hospital discharge

cases treated by the basal-bolus procedure ( $n=5,2.4 \%$, and $n=5,27.8 \%$, respectively; $P<0.001)$. The total amount of insulin administered during the first 6 days was higher in the presence of diabetes by history vs. newly diagnosed diabetes (on average $109.6 \pm 144.0$ IU vs. $44.7 \pm 65.3 ; P=0.06)$, also including on average $55 \pm 63 \mathrm{IU}$ by e.v. administration in 11 individuals of the former group and $50 \mathrm{IU}$ in 2 individuals of the latter group.

\section{Treatment Outcomes and Association with Stress Hyperglycemia}

One or more events were registered in 96 patients (25.4\%). The most severe and most common events are reported in Table 2, grouped by both hyperglycemic state and the presence of stress-induced hyperglycemia at entry. Forty-four patients died in hospital; the difference in fatality rates was borderline higher in patients without diabetes $(14.6 \%)$ compared with all cases with diabetes $(8.1 \% ; P=0.052)$ and nearly three times higher in patients with versus without stress hyperglycemia ( $15 \%$ vs. $5.3 \% ; P=0.003)$. No difference was present between insulin-treated and non-insulin-treated patients $(5.3 \%$ vs. $6.3 \%$; $P=0.747)$, irrespective of the presence of 
Table 2 In-hospital event rates according to the presence of diabetes and stress-induced hyperglycemia

\begin{tabular}{|c|c|c|c|c|c|c|c|}
\hline \multirow{2}{*}{$\begin{array}{l}\text { Event number } \\
\text { (percent) }\end{array}$} & \multicolumn{4}{|c|}{ Grouped by hyperglycemic state } & \multicolumn{3}{|c|}{ Grouped by stress hyperglycemia } \\
\hline & $\begin{array}{l}\text { No } \\
\text { diabetes } \\
(n=206)\end{array}$ & $\begin{array}{l}\text { Diabetes by } \\
\text { history } \\
(n=154)\end{array}$ & $\begin{array}{l}\text { Newly diagnosed } \\
\text { diabetes }(n=18)\end{array}$ & $P$ Value & $\begin{array}{l}\text { SHR }<1.2 \\
(n=132)\end{array}$ & $\begin{array}{l}\text { SHR } \geq 1.2 \\
(n=246)\end{array}$ & $P$ value \\
\hline All events & $56(27.2)$ & $44(28.6)$ & $5(27.8)$ & 0.959 & $23(17.3)$ & $82(33.5)$ & 0.007 \\
\hline In-hospital death & $30(14.6)$ & $13(8.4)$ & $1(5.6)$ & 0.143 & $7(5.3)$ & $37(15.0)$ & 0.005 \\
\hline $\begin{array}{l}\text { Time to } \\
\text { death (days)* }\end{array}$ & $7[10]$ & $9[7]$ & $33[-]$ & 0.186 & $7[7]$ & $8[9]$ & 0.797 \\
\hline \multicolumn{8}{|l|}{$\begin{array}{l}\text { Non-infectious } \\
\text { events }\end{array}$} \\
\hline Hypoglycemia & $4(1.9)$ & $16(10.4)$ & $1(5.6)$ & 0.002 & $9(6.8)$ & $12(4.9)$ & 0.432 \\
\hline $\begin{array}{l}\text { Acute } \\
\text { myocardial } \\
\text { infarction }\end{array}$ & $1(0.5)$ & $1(0.7)$ & $0-$ & 0.929 & $1(0.8)$ & $1(0.4)$ & 0.653 \\
\hline Heart failure & $36(17.6)$ & $40(26.1)$ & $3(16.7)$ & 0.128 & $25(19.1)$ & $54(22.0)$ & 0.509 \\
\hline $\begin{array}{l}\text { Cerebrovascular } \\
\text { events }\end{array}$ & $1(0.5)$ & $1(0.7)$ & $0-$ & 0.929 & $2(1.5)$ & $0-$ & 0.053 \\
\hline Arrythmias & $6(2.9)$ & $4(2.6)$ & $1(5.6)$ & 0.779 & $3(2.3)$ & $8(3.3)$ & 0.589 \\
\hline $\begin{array}{l}\text { Progressive renal } \\
\text { disease }^{\#}\end{array}$ & $9(4.4)$ & $7(4.5)$ & $2(11.2)$ & 0.430 & $4(3.0)$ & $14(5.7)$ & 0.247 \\
\hline Others & $26(12.6)$ & $27(17.5)$ & $4(22.2)$ & 0.299 & $16(12.1)$ & $41(16.7)$ & 0.292 \\
\hline $\begin{array}{l}\text { Systemic } \\
\text { infections }^{\circ}\end{array}$ & $\begin{array}{r}32 / 138 \\
(23.2)\end{array}$ & $27 / 111(24.3)$ & $3 / 11(27.3)$ & 0.944 & $\begin{array}{l}15 / 100 \\
(15.0)\end{array}$ & $\begin{array}{l}47 / 160 \\
(29.4)\end{array}$ & 0.011 \\
\hline
\end{tabular}

Note that more than one event might occur in individual patients

Data are presented as number of cases (\%)

*Median [interquartile range]

${ }^{\circ}$ In patients without infection at admission (total number, 260)

${ }^{\#}$ Renal function deterioration by $\geq 1 \mathrm{CKD}$ stage

diabetes or stress hyperglycemia. All-event rates were not associated with any hyperglycemic state, but were again more common in the presence of stress hyperglycemia $(33.5 \%$ vs. $17.3 ; P=0.007)$. No differences in non-infectious events were present, with the notable exclusion of hypoglycemic events (more common in insulin-treated patients with diabetes). Arrythmias were more common in insulin-treated cases and were associated with $\mathrm{K}^{+}$ levels < $3.6 \mathrm{mEq} / \mathrm{l}$ (OR 6.77; 95\% CI 1.47-31.4), without any relation with hypoglycemic events
(OR 4.07; 95\% CI 0.82-20.2) or death (OR 2.89; 95\% CI 0.76-11.7). No relation was demonstrated between hypoglycemic events and death rates (OR 1.28; 95\% CI 0.36-4.55). Other events included gastrointestinal and biliary events, as well as psychiatric disturbances, evenly distributed among groups.

The rate of newly developed infections was not different in relation to presence/absence of diabetes history or unknown diabetes, but was two times higher in the presence of stress hyperglycemia $(29.4 \%$ vs. $15.0 \% ; P=0.011)$ 
Table 3 Univariate and multivariate logistic regression analysis of factors associated with unfavorable outcomes in the whole population

\begin{tabular}{|c|c|c|c|c|}
\hline \multirow[t]{2}{*}{ Variable } & \multicolumn{2}{|c|}{ Univariate analysis } & \multicolumn{2}{|c|}{ Multivariate analysis } \\
\hline & $\overline{\text { OR }(95 \% \mathrm{CI})}$ & $P$ value & OR (95\% CI) & $P$ value \\
\hline \multicolumn{5}{|l|}{ In-hospital death } \\
\hline Age $(\times 10$ years $)$ & $2.42(1.57-3.73)$ & $<0.001$ & $2.33(1.50-3.60)$ & 0.002 \\
\hline Sex (male) & $0.82(0.43-1.54)$ & 0.531 & & \\
\hline BMI $\left(\mathrm{kg} / \mathrm{m}^{2}\right)$ & $1.01(0.88-1.17)$ & 0.845 & & \\
\hline Age-adjusted Charlson's score & $1.07(0.95-1.21)$ & 0.257 & & \\
\hline Glucose at entry $(\times 10 \mathrm{mg} / \mathrm{dl})$ & $1.03(0.99-1.06)$ & 0.113 & & \\
\hline Diabetes & $0.52(0.27-1.02)$ & 0.056 & $0.70(0.35-1.42)$ & 0.327 \\
\hline Stress hyperglycemia $(\mathrm{SHR} \geq 1.2)$ & $3.20(1.39-7.40)$ & 0.007 & $2.94(1.24-6.95)$ & 0.014 \\
\hline Insulin treatment & $1.13(0.60-2.12)$ & 0.711 & & \\
\hline Basal-bolus treatment* & $0.60(0.23-1.55)$ & 0.294 & & \\
\hline \multicolumn{5}{|l|}{ Complications (all events) } \\
\hline Age $(\times 10$ years $)$ & $1.41(1.14-1.75)$ & 0.002 & $1.44(1.15-1.81)$ & 0.013 \\
\hline Sex (male) & $0.85(0.54-1.34)$ & 0.490 & & \\
\hline BMI $\left(\mathrm{kg} / \mathrm{m}^{2}\right)$ & $1.05(0.96-1.14$ & 0.267 & & \\
\hline Age-adjusted Charlson's score & $1.06(0.97-1.17)$ & 0.215 & & \\
\hline Glucose at entry $(\times 10 \mathrm{mg} / \mathrm{dl})$ & $1.03(1.00-1.05)$ & 0.039 & $1.02(1.00-1.05)$ & 0.097 \\
\hline Diabetes & $1.07(0.68-1.68)$ & 0.778 & & \\
\hline Stress hyperglycemia $(\mathrm{SHR} \geq 1.2)$ & $2.41(1.43-4.06)$ & 0.001 & $2.07(1.19-3.59)$ & 0.010 \\
\hline Insulin treatment & $1.45(0.92-2.29)$ & 0.105 & & \\
\hline Basal-bolus treatment* & $0.64(0.34-1.23)$ & 0.182 & & \\
\hline \multicolumn{5}{|l|}{ Systemic infections ${ }^{\circ}$} \\
\hline Age $(\times 10$ years $)$ & $1.16(0.91-1.47)$ & 0.220 & & \\
\hline Sex (male) & $0.89(0.51-1.54)$ & 0.672 & & \\
\hline BMI $\left(\mathrm{kg} / \mathrm{m}^{2}\right)$ & $1.01(0.92-1.11)$ & 0.869 & & \\
\hline Age-adjusted Charlson's score & $1.03(0.93-1.15)$ & 0.548 & & \\
\hline Glucose at entry $(\times 10 \mathrm{mg} / \mathrm{dl})$ & $1.01(0.98-1.04)$ & 0.563 & & \\
\hline Diabetes & $1.15(0.67-1.98)$ & 0.618 & & \\
\hline Stress hyperglycemia $(\mathrm{SHR} \geq 1.2)$ & $2.36(1.24-4.50)$ & 0.009 & $2.36(1.24-4.50)$ & 0.009 \\
\hline Insulin treatment & $1.21(0.70-2.09)$ & 0.496 & & \\
\hline
\end{tabular}


Table 3 continued

\begin{tabular}{llllll}
\hline Variable & Univariate analysis & & & Multivariate analysis & \\
${$\cline { 2 - 3 }$(\mathbf{9 5 \%} \mathbf{C I})} }$ & $\boldsymbol{P}$ value & & OR (95\% CI $)$ & $\boldsymbol{P}$ value \\
\hline Basal-bolus treatment & $0.61(0.28-1.30)$ & 0.198 & & \\
\hline
\end{tabular}

${ }^{*}$ Limited to insulin-treated patients

${ }^{\circ}$ In patients without infection at admission $(n=260)$

after exclusion of cases with infections at admission.

The results of logistic regression analysis are reported in Table 3. At univariate analysis, only stress hyperglycemia was associated with all tested outcomes (in-hospital death, all complications and newly developed infections), particularly in the absence of diabetes and in noninsulin-treated cases (Fig. 3). The association with in-hospital death was stronger in subjects

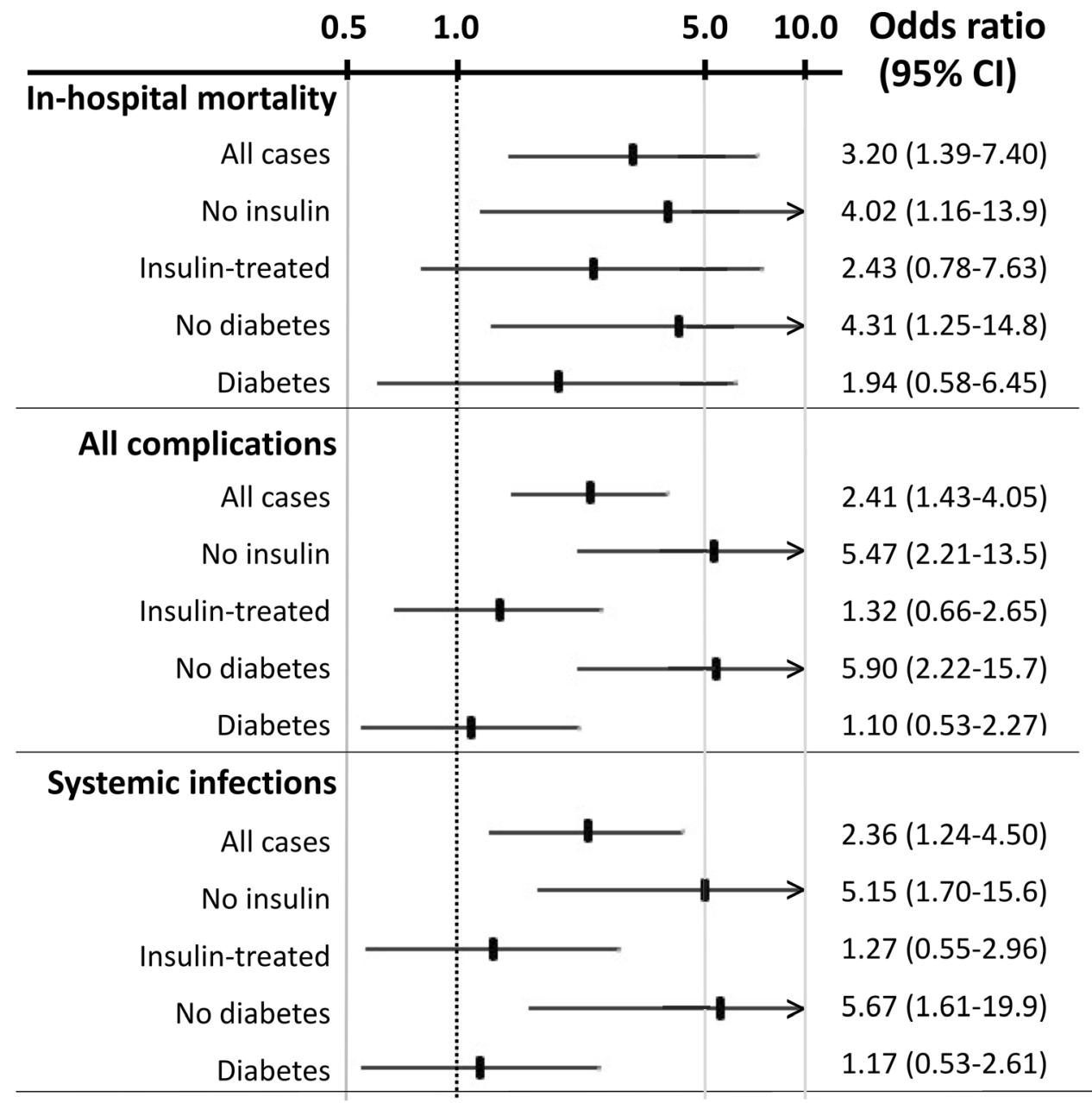

Fig. 3 Graphical presentation of the risk for in-hospital mortality and complicating events associated with stress hyperglycemia in relation to insulin treatment and the

presence/absence of diabetes. Data are derived from univariate logistic regression analysis 
without diabetes (OR 4.31, 95\% CI 1.25-14.81) and in non-insulin-treated patients (4.02, 95\% CI 1.16-13.92). Similarly, the association of stress hyperglycemia with all complications and with systemic infections was maintained in non-insulin-treated cases (OR 5.47, 95\% CI 2.21-13.52, and OR 5.15, 95\% CI 1.70-15.62, respectively) as well as in patients without diabetes (OR 5.90, 95\% CI 2.22-15.71, and OR 5.67, 95\% CI 1.61-19.92).

Age, diabetes and glucose levels at admission were the other variables that entered the multivariable regression analysis for death and complications; at the end of the procedure, only stress hyperglycemia and age remained in the regression. In the multivariable analysis, the risk of death and of all-event rates associated with stress hyperglycemia was particularly high in subjects without diabetes (OR 3.95, 95\% CI 1.12-13.86 and OR 5.70, 95\% CI 2.12-15.32, respectively), as was the risk in non-insulintreated cases (OR 3.08, 95\% CI 1.56-6.06 and OR 5.45, 95\% CI 2.18-13.53, respectively).

No significant differences in outcomes were observed in insulin-treated subjects according to the insulin delivery strategy (basal-bolus vs. sliding scale), although for most events a trend was observed in favor of the basal-bolus procedure (OR 0.60, 95\% CI 0.23-1.55; OR 0.65, 95\% CI $0.34-1.62$ and OR $0.61,95 \%$ CI $0.28-1.30$ for death, all events and infections, respectively). The relation of outcomes with stress hyperglycemia was not significantly modified by adjustment for factors potentially affecting HbA1c levels: advanced chronic kidney disease (CKD grade $3 \mathrm{~b}$ or higher), anemia (hemoglobin levels $<10 \mathrm{~g} / \mathrm{dl}$ ) and the presence of cirrhosis (Supplementary Table 1).

The sensitivity analysis using the cut-offs of 1.14 and 1.26 for the definition of stress hyperglycemia confirmed the results reported above (Supplementary Table 2).

\section{DISCUSSION}

The study confirms the importance of stressrelated hyperglycemia for the development of poor outcomes in individuals admitted to internal/geriatric units for acute events or exacerbation of pre-existing diseases. The risk of death, as well as the risk of complications, was higher in the presence of stress hyperglycemia than in diabetes per se and extended to subjects in whom stress hyperglycemia added to the hyperglycemia of diabetes, either well known and treated or newly diagnosed and untreated. The proportion of cases diagnosed with stress hyperglycemia was particularly high in the categories without previously diagnosed diabetes, although the cut-off of relative hyperglycemia was set at a value (1.20) higher than in previous studies $[6,7]$, considering uncertainty about appropriate fasting in a few cases. The relatively less severity of stress hyperglycemia in individuals with diabetes is in keeping with the importance of the modulatory effect of glucoselowering treatment on hyperglycemia. In the absence of diabetes, as well as in undiagnosed diabetes, stress hyperglycemia is unopposed by treatment and the resulting hyperglycemia carries an additional risk of adverse events.

The negative role of stress hyperglycemia is well known. In both patients with and without diabetes and ST elevation myocardial infarction, stress hyperglycemia ratio, defined by SHR quartiles, was a useful predictive marker of major adverse, cardiovascular and cerebrovascular events (including death)[30]. Among patients admitted for ischemic stroke in four Italian hospitals who developed parenchymal hematoma after thrombolysis, hyperglycemia at admission predicted death and permanent disability in a linear, glucose-dependent response [23]. Similar data were obtained in an interim analysis of the present population, limited to patients with ischemic stroke, where stress hyperglycemia increased the risk of hemorrhagic transformation [31].

In a multicenter study in an Internal Medicine setting, mean blood glucose on the day of admission was an independent negative prognostic factor, suggesting that prompt treatment of hyperglycemia is needed to improve hospital outcomes [1]. In trauma patients, blood glucose at admission predicted mortality and infections $[6,32,33]$, which were reduced by achieving normoglycemia in the early post-traumatic period [34]. An analysis carried out in emergency departments confirmed the negative role 
of stress hyperglycemia also in the presence of diabetes, with sepsis-repeated mortality increasing over five times for a SHR $\geq 1.14$ [7]. Very recently, the importance of hyperglycemia has been associated with poor outcomes in patients infected with COVID-19, where newly diagnosed diabetes and admission hyperglycemia predicted COVID-19 severity and more rapid respiratory deterioration better than the history of diabetes [5].

In other settings, the risk of systemic infections was independently related to hyperglycemia, as well as to stress hyperglycemia, as demonstrated by an extensive metaanalysis [35]. In the present study, we confirmed the prognostic significance of stress hyperglycemia for newly developed infections; the risk was higher than that carried out by glucose at entry, despite prompt correction of hyperglycemia in most cases, both with insulin or by oral agents $(n=76)$.

Whatever the origin of hyperglycemia, our program was intended to achieve a prompt control of hyperglycemia, and a glucose target between 120 and $150 \mathrm{mg} / \mathrm{dl}$ was originally planned, at least in the first few days of admission. Despite insulin administration-and e.v. insulin in a non-negligible proportion of cases-the planned target was not reached before hospital discharge or death in a significant proportion of cases, particularly in the presence of diabetes. There is considerable debate regarding the desired glucose target for in-hospital patients. Guidelines of the American Diabetes Association suggest a glucose range of $140-180 \mathrm{mg} / \mathrm{dl}$ in critically ill cases and lower values of 110-140 for selected patients, as long as they can be achieved without significant risk of hypoglycemia [14]. In our cohort, insulin treatment was definitely associated with a few hypoglycemic events, possibly facilitated by insulin-dependent low potassium levels, but the rare events did not carry an additional risk of death or arrithmya. It would be important to test the possible advantage of glucose control by the newly available glucose-lowering drugs on both safety and effectiveness in a larger population.

Insulin treatment was intended to modulate the systemic inflammatory response and immune cells, activated in severe trauma, burn injury and sepsis [36], via possible modulation of glucose transporters [37], and several studies in these areas confirm that tailored insulin administration reduced the risk of local or systemic infections $[35,38,39]$. In the present study we could not reduce the risk of newly developed infections and did not confirm the superiority of the basal-bolus procedure vs. the sliding-scale regimen on infections previously documented in a surgical setting [17]. The basalbolus regimen reduced the risk of outcomes in univariate analysis only to an insignificant extent; negative results might be explained by the very high number of events occurring in the first few days after admission, before the more accurate insulin modulation of glucose disposal might have produced beneficial effects.

The study has limitations that deserve discussion. First, the population was highly heterogeneous, which may limit the external validation of the results. This was however part of the original aim of the study, i.e., to test the significance of stress hyperglycemia and its rapid control in a general medicine/geriatric setting. A second limitation is the use of SHR as a correlate of stress hyperglycemia, a tool not universally accepted. The number of cases identified as stress hyperglycemia, in both the presence and absence of diabetes, was very high; sensitivity analyses show that data are however stable, independent of the cut-offs for stress hyperglycemia and adjustments for potential confounders, confirming the validity of the ratio. Finally, the sample size was however insufficient to achieve solid data on treatment strategies.

\section{CONCLUSION}

In summary, the study confirms that stress-related hyperglycemia, a protective response providing fuel for the immune system and the brain at time of stress, might become potentially deleterious [12], possibly contributing to adverse outcomes. It remains to be determined whether a direct cause-effect relation exists or the severity and duration of 
hyperglycemia simply reflect more severe whole-body stress.

\section{ACKNOWLEDGEMENTS}

Funding. FA Barbanti is supported by a research contract financed by Italian Ministry of Health and Italian Regions (NET-201602364191). No funding or sponsorship was received for the publication of this article.

Authorship. All named authors meet the International Committee of Medical Journal Editors (ICMJE) criteria for authorship for this article, take responsibility for the integrity of the work as a whole, and have given their approval for this version to be published.

Author Contribution. All authors contributed to the study conception and design. Material preparation and data collection were performed by $\mathrm{R}$ Di Luzio and R Dusi. Data analysis was carried out by $\mathrm{G}$ Marchesini. The first draft of the manuscript was written by $G$ Marchesini, FA Barbanti and G Bianchi and all authors commented on previous versions of the manuscript. All authors read and approved the final manuscript.

Disclosures. Raffaella Di Luzio, Rachele Dusi, Francesca Alessandra Barbanti, Pietro Calogero and Giampaolo Bianchi have nothing to disclose in relation to the present study. Giulio Marchesini participated in advisory boards of Gilead, Novartis, MSD, Pfizer, and Astra-Zeneca.

Compliance with Ethics Guidelines. The protocol was approved by the ethics committee, Sant'Orsola-Malpighi Hospital (protocol $27 / 2017 /$ U/Oss and amendments), and by the ethics committee of the Local Health Unit of Bologna (CE-BI 17044). All patients signed an informed consent to participation and data upload. This study was performed in accordance with the Helsinki Declaration of 1964 and its later amendments.
Data Availability. The datasets generated during and/or analyzed during the current study are available from the corresponding author on reasonable request.

Open Access. This article is licensed under a Creative Commons Attribution-NonCommercial 4.0 International License, which permits any non-commercial use, sharing, adaptation, distribution and reproduction in any medium or format, as long as you give appropriate credit to the original author(s) and the source, provide a link to the Creative Commons licence, and indicate if changes were made. The images or other third party material in this article are included in the article's Creative Commons licence, unless indicated otherwise in a credit line to the material. If material is not included in the article's Creative Commons licence and your intended use is not permitted by statutory regulation or exceeds the permitted use, you will need to obtain permission directly from the copyright holder. To view a copy of this licence, visit http://creativecommons.org/licenses/by$\mathrm{nc} / 4.0 /$.

\section{REFERENCES}

1. Avanzini F, Mafrici A, Riva E, et al. A multicenter observational study on the management of hyperglycemia in patients with acute coronary syndrome. Nutr Metab Cardiovasc Dis. 2015;25:916-23.

2. Pieralli F, Bazzini C, Fabbri A, et al. The classification of hospitalized patients with hyperglycemia and its implication on outcome: results from a prospective observational study in internal medicine. Intern Emerg Med. 2016;11:649-56.

3. Seghieri G, Policardo L, Profili F, Francesconi P, Anichini R, Del Prato S. Hospital incidental diagnosis of diabetes: a population study. J Diabetes Complicat. 2016;30:457-61.

4. Sganga F, Landi F, Vetrano DL, et al. Impact of hospitalization on modification of drug regimens: results of the criteria to assess appropriate medication use among elderly complex patients study. Geriatr Gerontol Int. 2016;16:593-9.

5. Fadini GP, Morieri ML, Boscari F, et al. Newly-diagnosed diabetes and admission hyperglycemia predict COVID-19 severity by aggravating 
respiratory deterioration. Diabetes Res Clin Pract. 2020;168:108374.

6. Di Luzio R, Dusi R, Mazzotti A, Petroni ML, Marchesini G, Bianchi G. Stress hyperglycemia and complications following traumatic injuries in individuals with/without diabetes: the case of orthopedic surgery. Diabetes Metab Syndr Obes. 2020;13: 9-17.

7. Fabbri A, Marchesini G, Benazzi B, et al. Stress hyperglycemia and mortality in subjects with diabetes and sepsis. Crit Care Expl. 2020;2:e0152.

8. Harp JB, Yancopoulos GD, Gromada J. Glucagon orchestrates stress-induced hyperglycaemia. Diabetes Obes Metab. 2016;18:648-53.

9. Esposito K, Nappo F, Marfella R, et al. Inflammatory cytokine concentrations are acutely increased by hyperglycemia in humans: role of oxidative stress. Circulation. 2002;106:2067-72.

10. Losser MR, Damoisel C, Payen D. Bench-to-bedside review: glucose and stress conditions in the intensive care unit. Crit Care. 2010;14:231.

11. Capes SE, Hunt D, Malmberg K, Pathak P, Gerstein HC. Stress hyperglycemia and prognosis of stroke in nondiabetic and diabetic patients: a systematic overview. Stroke. 2001;32:2426-32.

12. Chen Y, Yang X, Meng K, et al. Stress-induced hyperglycemia after hip fracture and the increased risk of acute myocardial infarction in nondiabetic patients. Diabetes Care. 2013;36:3328-32.

13. Umpierrez GE, Isaacs SD, Bazargan N, You X, Thaler LM, Kitabchi AE. Hyperglycemia: an independent marker of in-hospital mortality in patients with undiagnosed diabetes. J Clin Endocrinol Metab. 2002;87:978-82.

14. American Diabetes Association. 15. Diabetes care in the hospital: standards of medical care in diabetes2021. Diabetes Care. 2021;44:S211-20.

15. Associazione Medici Diabetologi (AMD), Società Italiana di Diabetologia (SID). Standard Italiani per la Cura del Diabete. In: AMD, SID, editors; 2018.

16. Gallagher JM, Erich RA, Gattermeyer R, Beam KK. Postoperative hyperglycemia can be safely and effectively controlled in both diabetic and nondiabetic patients with use of a subcutaneous insulin protocol. JB JS Open Access. 2017;2:e0008.

17. Di Luzio R, Dusi R, Morigi A, et al. Nurse-managed basal-bolus versus sliding-scale insulin regimen in subjects with hyperglycemia at admission for orthopedic surgery: a propensity score approach. Acta Diabetol. 2020;57:835-42.
18. Akiboye F, Rayman G. Management of hyperglycemia and diabetes in orthopedic surgery. Curr Diab Rep. 2017;17:13.

19. Charlson ME, Pompei P, Ales KL, MacKenzie CR. A new method of classifying prognostic comorbidity in longitudinal studies: development and validation. J Chronic Dis. 1987;40:373-83.

20. Nathan DM, Kuenen J, Borg R, et al. Translating the A1C assay into estimated average glucose values. Diabetes Care. 2008;31:1473-8.

21. Roberts GW, Quinn SJ, Valentine N, et al. Relative hyperglycemia, a marker of critical ilness: introducing the stress hyperglycemia ratio. J Clin Endocrinol Metab. 2015;100:4490-7.

22. Peralta G, Sanchez MB, Garrido JC, et al. Altered blood glucose concentration is associated with risk of death among patients with community-acquired Gram-negative rod bacteremia. BMC Infect Dis. 2010;10:181.

23. Paciaroni M, Agnelli G, Caso V, et al. Acute hyperglycemia and early hemorrhagic transformation in ischemic stroke. Cerebrovasc Dis. 2009;28:119-23.

24. Suidan RS, Leitao MM Jr, Zivanovic O, et al. Predictive value of the Age-Adjusted Charlson Comorbidity Index on perioperative complications and survival in patients undergoing primary debulking surgery for advanced epithelial ovarian cancer. Gynecol Oncol. 2015;138:246-51.

25. McCowen KC, Malhotra A, Bistrian BR. Stress-induced hyperglycemia. Crit Care Clin. 2001;17: 107-24.

26. Christman AL, Lazo M, Clark JM, Selvin E. Low glycated hemoglobin and liver disease in the U.S. population. Diabetes Care. 2011;34:2548-50.

27. Lo C, Lui M, Ranasinha S, et al. Defining the relationship between average glucose and HbA1c in patients with type 2 diabetes and chronic kidney disease. Diabetes Res Clin Pract. 2014;104:84-91.

28. Nadelson J, Satapathy SK, Nair S. Glycated hemoglobin levels in patients with decompensated cirrhosis. Int J Endocrinol. 2016;2016:8390210.

29. Levey AS, Stevens LA. Estimating GFR using the CKD Epidemiology Collaboration (CKD-EPI) creatinine equation: more accurate GFR estimates, lower CKD prevalence estimates, and better risk predictions. Am J Kidney Dis. 2010;55:622-7.

30. Yang Y, Kim TH, Yoon KH, et al. The stress hyperglycemia ratio, an index of relative hyperglycemia, as a predictor of clinical outcomes after 
percutaneous coronary intervention. Int J Cardiol. 2017;241:57-63.

31. Dusi R, Marcialis C, Di Luzio R, Zoli M, Bianchi G. L'iperglicemia da stress come possibile predittore di peggior outcome nel paziente ricoverato per ictus. In: $64^{\circ}$ Congresso Nazionale SIGG. Pisa, Rome: Pacini ditore; 2019. p. 156.

32. Bochicchio GV, Salzano L, Joshi M, Bochicchio K, Scalea TM. Admission preoperative glucose is predictive of morbidity and mortality in trauma patients who require immediate operative intervention. Am Surg. 2005;71:171-4.

33. Richards JE, Kauffmann RM, Obremskey WT, May AK. Stress-induced hyperglycemia as a risk factor for surgical-site infection in nondiabetic orthopedic trauma patients admitted to the intensive care unit. J Orthop Trauma. 2013;27:16-21.

34. Scalea TM, Bochicchio GV, Bochicchio KM, Johnson SB, Joshi M, Pyle A. Tight glycemic control in critically injured trauma patients. Ann Surg. 2007;246:605-10 (discussion 610-602).
35. Martin ET, Kaye KS, Knott C, et al. Diabetes and risk of surgical site infection: a systematic review and meta-analysis. Infect Control Hosp Epidemiol. 2016;37:88-99.

36. Deng HP, Chai JK. The effects and mechanisms of insulin on systemic inflammatory response and immune cells in severe trauma, burn injury, and sepsis. Int Immunopharmacol. 2009;9:1251-9.

37. Dungan KM, Braithwaite SS, Preiser JC. Stress hyperglycaemia. Lancet. 2009;373:1798-807.

38. Chrastil J, Anderson MB, Stevens V, Anand R, Peters $\mathrm{CL}$, Pelt CE. Is hemoglobin A1c or perioperative hyperglycemia predictive of periprosthetic joint infection or death following primary total joint arthroplasty? J Arthroplasty. 2015;30:1197-202.

39. Mraovic B, Suh D, Jacovides C, Parvizi J. Perioperative hyperglycemia and postoperative infection after lower limb arthroplasty. J Diabetes Sci Technol. 2011;5:412-8. 